\title{
Congruences for the coefficients of quotients of Eisenstein series
}

by

Bruce C. Berndt and Ae Ja Yee (Urbana, IL)

1. Introduction. Ramanujan, in [6], [7, pp. 232-238], established the following famous congruences for $p(n)$, the number of partitions of $n$ :

$$
\begin{aligned}
p(5 n+4) & \equiv 0(\bmod 5), \\
p(7 n+5) & \equiv 0(\bmod 7), \\
p(11 n+6) & \equiv 0(\bmod 11) .
\end{aligned}
$$

In calculating the coefficients of certain quotients of the Eisenstein series

$$
\begin{aligned}
& P(q):=1-24 \sum_{k=1}^{\infty} \frac{k q^{k}}{1-q^{k}}=1-24 \sum_{n=1}^{\infty} \sigma(n) q^{n}, \\
& Q(q):=1+240 \sum_{k=1}^{\infty} \frac{k^{3} q^{k}}{1-q^{k}}=1+240 \sum_{n=1}^{\infty} \sigma_{3}(n) q^{n}, \\
& R(q):=1-504 \sum_{k=1}^{\infty} \frac{k^{5} q^{k}}{1-q^{k}}=1-504 \sum_{n=1}^{\infty} \sigma_{5}(n) q^{n},
\end{aligned}
$$

studied in [1] and [2], where $|q|<1$, we noticed that for some quotients of Eisenstein series the coefficients in certain arithmetic progressions are divisible by prime powers, usually a power of 3 . In view of Ramanujan's famous congruences for $p(n)$, it seemed natural for us to systematically investigate congruences of this type for Eisenstein series. In some cases, it was very easy to establish our observations, but in other cases, the task was considerably more difficult.

We summarize our findings in the table below. In general, write $F(q):=$ $\sum_{n=0}^{\infty} \alpha_{n} q^{n}$.

2000 Mathematics Subject Classification: Primary 11F30.

Research of B. C. Berndt partially supported by grant MDA904-00-1-0015 from the National Security Agency.

Research of A. J. Yee partially supported by a grant from the Number Theory Foundation. 


\begin{tabular}{|c|c|c|}
\hline$F(q)$ & $n \equiv 2(\bmod 3)$ & $n \equiv 4(\bmod 8)$ \\
\hline $1 / P(q)$ & $\alpha_{n} \equiv 0\left(\bmod 3^{4}\right)$ & \\
\hline $1 / Q(q)$ & $\alpha_{n} \equiv 0\left(\bmod 3^{2}\right)$ & \\
\hline $1 / R(q)$ & $\alpha_{n} \equiv 0\left(\bmod 3^{3}\right)$ & $\alpha_{n} \equiv 0\left(\bmod 7^{2}\right)$ \\
\hline$P(q) / Q(q)$ & $\alpha_{n} \equiv 0\left(\bmod 3^{3}\right)$ & \\
\hline$P(q) / R(q)$ & $\alpha_{n} \equiv 0\left(\bmod 3^{2}\right)$ & $\alpha_{n} \equiv 0(\bmod 7)$ \\
\hline$Q(q) / R(q)$ & $\alpha_{n} \equiv 0\left(\bmod 3^{3}\right)$ & \\
\hline$P^{2}(q) / R(q)$ & $\alpha_{n} \equiv 0\left(\bmod 3^{5}\right)$ & \\
\hline
\end{tabular}

To prove our observations, we need to carefully examine $\sigma_{k}(n)$, the sum of the $k$ th powers of the divisors of the positive integer $n$, for odd $k$. In Section 2 , we calculate $\sigma_{k}(n)$ for $n \equiv 2(\bmod 3)$, and state congruences and equalities for $\sigma_{k}(n)$ established by D. B. Lahiri in [4] and [5]. In Section 3, congruences for the coefficients of $1 / Q(q), 1 / R(q), P(q) / Q(q), P(q) / R(q)$, and $Q(q) / R(q)$ are proved very easily. We show the congruences for the coefficients of $1 / P(q)$ and $P^{2}(q) / R(q)$ in Sections 4 and 5 , respectively.

2. Preliminaries. In what follows, let $k$ be an odd positive integer, and we write $\sigma(n)$ for $\sigma_{1}(n)$. We examine $\sigma_{k}\left(p^{r}\right)$, where $p$ is a prime. We consider 3 cases: (i) $p=3 x-1$ and $r$ is odd, (ii) $p=3 x-1$ and $r$ is even, and (iii) $p=3 x+1$.

CASE (i): $p=3 x-1$ and $r$ is odd. It follows easily from elementary considerations below that

$$
\sigma_{k}\left(p^{r}\right) \equiv 0(\bmod 3)
$$

Moreover,

$$
\sigma_{3}\left(p^{r}\right) \equiv 0\left(\bmod 3^{2}\right) .
$$

However, we need a more refined congruence in some of our applications. To that end, write

$$
\begin{aligned}
\sigma_{k}\left(p^{r}\right) & =1+p^{k}+\ldots+p^{r k} \\
& =\left(1+p^{k}\right)\left(1+p^{2 k}+\ldots+p^{(r-1) k}\right) \\
& \equiv\left(1+(3 x-1)^{k}\right)\left(a_{k}+3 b_{k}+3^{2} c_{k}\right)\left(\bmod 3^{4}\right),
\end{aligned}
$$

where

$$
\begin{aligned}
& a_{k}:=\sum_{j=0}^{(r-1) / 2}(-1)^{2 j k}=\frac{r+1}{2}, \\
& b_{k}:=\sum_{j=0}^{(r-1) / 2}(-1)^{2 j k-1}\left(\begin{array}{c}
2 j k \\
1
\end{array}\right) x=-x \frac{r^{2}-1}{4} k,
\end{aligned}
$$


(2.6) $c_{k}:=\sum_{j=0}^{(r-1) / 2}(-1)^{2 j k-2}\left(\begin{array}{c}2 j k \\ 2\end{array}\right) x^{2}=\frac{\left(r^{2}-1\right) r}{12} x^{2} k^{2}-\frac{r^{2}-1}{8} x^{2} k$

$$
=: u k^{2}+v k \text {. }
$$

CASE (ii): $p=3 x-1$ and $r$ is even. Recall that $k$ is odd. Then

$$
\sigma_{k}\left(p^{r}\right)=1+p^{k}+\ldots+p^{r k} \equiv\left(A_{k}+3 B_{k}+3^{2} C_{k}\right)\left(\bmod 3^{3}\right),
$$

where

$$
\begin{aligned}
A_{k} & :=\sum_{j=0}^{r}(-1)^{j k}=1 \\
B_{k} & :=\sum_{j=0}^{r}(-1)^{j k-1}\left(\begin{array}{c}
j k \\
1
\end{array}\right) x=-\frac{r}{2} x k \\
C_{k} & :=\sum_{j=0}^{r}(-1)^{j k-2}\left(\begin{array}{c}
j k \\
2
\end{array}\right) x^{2} \\
& =\left(\sum_{j=0}^{r}(-1)^{j} \frac{j^{2}}{2} x^{2}\right) k^{2}-\left(\sum_{j=0}^{r}(-1)^{j} \frac{j}{2} x^{2}\right) k=: u_{k} k^{2}+v_{k} k .
\end{aligned}
$$

CASE (iii) $: p=3 x+1$. Then

$$
\sigma_{k}\left(p^{r}\right)=1+p^{k}+\ldots+p^{r k} \equiv\left(A_{k}+3 B_{k}+3^{2} C_{k}\right)\left(\bmod 3^{3}\right)
$$

where

$$
\begin{aligned}
A_{k} & :=\sum_{j=0}^{r} 1=r+1 \\
B_{k} & :=\sum_{j=0}^{r}\left(\begin{array}{c}
j k \\
1
\end{array}\right) x=\frac{r(r+1)}{2} x k \\
C_{k} & :=\sum_{j=0}^{r}\left(\begin{array}{c}
j k \\
2
\end{array}\right) x^{2} \\
& =\frac{r(r+1)(2 r+1)}{12} x^{2} k^{2}-\frac{r(r+1)}{4} x^{2} k=: u_{k} k^{2}+v_{k} k .
\end{aligned}
$$

We need a congruence for $\sigma_{k}(n)$ for $n \equiv 2(\bmod 3)$. There is at least one prime factor $p$ of $n$ such that $p \equiv 2(\bmod 3)$, and the maximum power of $p$ is odd for any $n \equiv 2(\bmod 3)$. Let $n=p^{r} p_{1}^{r_{1}} p_{2}^{r_{2}} \ldots p_{m}^{r_{m}}$, where $p \equiv 2(\bmod 3)$, $r$ is odd, and $p_{1}^{r_{1}} p_{2}^{r_{2}} \ldots p_{m}^{r_{m}} \equiv 1(\bmod 3)$. Since $\sigma_{k}$ is multiplicative, we see by $(2.1)$ and $(2.2)$ that

$$
\sigma_{k}(n) \equiv 0(\bmod 3) \quad \text { and } \quad \sigma_{3}(n) \equiv 0\left(\bmod 3^{2}\right) .
$$


Now, we consider $\sigma_{k}\left(p_{1}^{r_{1}} p_{2}^{r_{2}} \ldots p_{m}^{r_{m}}\right)$. Let

$$
\sigma_{k}\left(p_{i}^{r_{i}}\right): \equiv A_{k i}+3 B_{k i}+3^{2} C_{k i}\left(\bmod 3^{3}\right),
$$

for $i=1, \ldots, m$, and set, as in (2.10) or (2.14), $C_{k i}=u_{k i} k^{2}+v_{k i} k$. Then

$$
\begin{aligned}
\sigma_{k}\left(p_{1}^{r_{1}} p_{2}^{r_{2}} \ldots p_{m}^{r_{m}}\right) & =\sigma_{k}\left(p_{1}^{r_{1}}\right) \sigma_{k}\left(p_{2}^{r_{2}}\right) \ldots \sigma_{k}\left(p_{m}^{r_{m}}\right) \\
& \equiv \widehat{A}_{k}+3 \widehat{B}_{k}+3^{2} \widehat{C}_{k}\left(\bmod 3^{3}\right),
\end{aligned}
$$

where

$$
\begin{aligned}
& \widehat{A}_{k}:=\prod_{i=1}^{m} A_{k i}, \\
& \widehat{B}_{k}:=\widehat{A}_{k} \sum_{i=1}^{m} \frac{B_{k i}}{A_{k i}}, \\
& \widehat{C}_{k}:=\widehat{A}_{k} \sum_{i=1}^{m} \frac{C_{k i}}{A_{k i}}+\widehat{A}_{k} \sum_{i=1}^{m} \sum_{\substack{j=1 \\
j \neq i}}^{m} \frac{B_{k i} B_{k j}}{A_{k i} A_{k j}} .
\end{aligned}
$$

Then we easily see by elementary calculations on $\sigma_{k}\left(p_{i}^{r_{i}}\right)$ that

$$
\begin{aligned}
& \widehat{A}_{k}=\widehat{A}_{1}, \\
& \widehat{B}_{k}=k \widehat{B}_{1}, \\
& \widehat{C}_{k}=k^{2} U+k V,
\end{aligned}
$$

where

$$
U=\widehat{A}_{1} \sum_{i=1}^{m} \frac{u_{1 i}}{A_{1 i}}+\widehat{A}_{1} \sum_{i=1}^{m} \sum_{\substack{j=1 \\ j \neq i}}^{m} \frac{B_{1 i} B_{1 j}}{A_{1 i} A_{1 j}} \quad \text { and } \quad V=\widehat{A}_{1} \sum_{i=1}^{m} \frac{v_{1 i}}{A_{1 i}} .
$$

Necessary for our proofs are certain identities and congruences for $\sigma_{k}(n)$. Before stating them, recall that Ramanujan's tau function $\tau(n)$ is defined by

$$
\sum_{n=1}^{\infty} \tau(n) q^{n}:=q \prod_{n=1}^{\infty}\left(1-q^{n}\right)^{24} \quad \text { for }|q|<1 .
$$

Lahiri [4], [5] established many identities and congruences for $\sigma_{k}(n)$ and $\tau(n)$. Among them we state the identities and congruences we use in the remainder of the paper. Thus,

$$
2^{2} \cdot 3 \sum_{k=1}^{n-1} \sigma(k) \sigma(n-k)=5 \sigma_{3}(n)-(6 n-1) \sigma(n),
$$

(2.26) $\quad 2^{6} \cdot 3 \sum_{k_{1}+k_{2}=1}^{n-1} \sigma\left(k_{1}\right) \sigma\left(k_{2}\right) \sigma\left(n-k_{1}-k_{2}\right)$

$$
=7 \sigma_{5}(n)+(10-30 n) \sigma_{3}(n)+\left(1-12 n+24 n^{2}\right) \sigma(n),
$$




$$
\begin{gathered}
2^{4} \cdot 3^{2} \cdot 5 \cdot 7 \sum_{k=1}^{n-1} \sigma_{3}(k) \sigma_{5}(n-k)=11 \sigma_{9}(n)-3 \cdot 7 \sigma_{5}(n)+2 \cdot 5 \sigma_{3}(n) \\
2^{3} \cdot 3^{2} \cdot 7 \sum_{k=1}^{n-1} k \sigma(k) \sigma_{5}(n-k)=5 n \sigma_{7}(n)-2 \cdot 3 n^{2} \sigma_{5}(n)+n \sigma(n) \\
2^{2} \cdot 3^{2} \cdot 7 \cdot 691 \sum_{k=1}^{n-1} \sigma_{5}(k) \sigma_{5}(n-k) \\
=-2^{2} \cdot 3^{3} \cdot 7 \tau(n)+5 \cdot 13 \sigma_{11}(n)+691 \sigma_{5}(n) \\
-2^{2} \cdot 3^{3} \cdot 7 \tau(n)+5 \cdot 13 \sigma_{11}(n) \equiv 691\left\{20 \sigma_{7}(n)-2(21 n-10) \sigma_{5}(n)\right. \\
\left.\quad-105 \sigma_{3}(n)+2(63 n-10) \sigma(n)\right\}\left(\bmod 2^{4} \cdot 3^{4} \cdot 5^{2} \cdot 7 \cdot 691\right)
\end{gathered}
$$

The most thorough examination of divisor sum identities like those in (2.25)-(2.29) has been given by J. G. Huard, Z. M. Ou, B. K. Spearman, and K. S. Williams in [3].

By combining (2.29) and (2.30), we obtain

$$
\begin{aligned}
2^{2} \cdot 3^{2} \cdot 7 \sum_{k=1}^{n-1} \sigma_{5}(k) \sigma_{5}(n-k) \equiv 20 \sigma_{7}(n)-2(21 n-10) \sigma_{5}(n) \\
\quad-105 \sigma_{3}(n)+2(63 n-10) \sigma(n)+\sigma_{5}(n)\left(\bmod 2^{4} \cdot 3^{4} \cdot 5^{2} \cdot 7\right) .
\end{aligned}
$$

We need more congruences, which are found in [4] and [5], namely,

$$
\begin{aligned}
& n \sigma_{7}(n) \equiv 14 n \sigma_{5}(n)-\left(24 n^{2}-11 n\right) \sigma_{3}(n)\left(\bmod 2^{5} \cdot 3^{2} \cdot 5\right) \\
& 11 \sigma_{9}(n) \equiv 50(30 n-2) \sigma_{7}(n)-30\left(24 n^{2}-28 n+7\right) \sigma_{5}(n) \\
& \quad+20\left(72 n^{3}-108 n^{2}+45 n-5\right) \sigma_{3}(n) \\
& \quad-\left(864 n^{4}-1440 n^{3}+720 n^{2}-120 n+5\right) \sigma(n)\left(\bmod 2^{12} \cdot 3^{4}\right)
\end{aligned}
$$

3. Coefficients of $1 / R, 1 / Q, P / Q, P / R$, and $Q / R$. In this section, we show that the coefficient of $q^{n}$ in $1 / R(q)$ is divisible by $3^{3}$ and $7^{2}$ for $n \equiv 2(\bmod 3)$ and $n \equiv 4(\bmod 8)$, respectively. Since the proofs of the assertions for $1 / Q(q), P(q) / Q(q), P(q) / R(q)$, and $Q(q) / R(q)$ are similar, we omit them.

Theorem 3.1. In each case, set $F(q)=\sum_{n=0}^{\infty} \alpha_{n} q^{n},|q|<1$. Let $n \equiv$ $2(\bmod 3)$.

(a) If $F(q)=1 / R(q)$, then $\alpha_{n} \equiv 0\left(\bmod 3^{3}\right)$;

(b) if $F(q)=1 / Q(q)$, then $\alpha_{n} \equiv 0\left(\bmod 3^{2}\right)$;

(c) if $F(q)=P(q) / Q(q)$, then $\alpha_{n} \equiv 0\left(\bmod 3^{3}\right)$;

(d) if $F(q)=P(q) / R(q)$, then $\alpha_{n} \equiv 0\left(\bmod 3^{2}\right)$;

(e) if $F(q)=Q(q) / R(q)$, then $\alpha_{n} \equiv 0\left(\bmod 3^{3}\right)$. 
Let $n \equiv 4(\bmod 8)$.

(f) If $F(q)=1 / R(q)$, then $\alpha_{n} \equiv 0\left(\bmod 7^{2}\right)$;

(g) if $F(q)=P(q) / R(q)$, then $\alpha_{n} \equiv 0(\bmod 7)$.

Proof of (a). For sufficiently small $|q|$, from (1.3), we consider the geometric series expansion of $1 / R(q)$. Then

$$
\begin{aligned}
\frac{1}{R(q)} & =1+504 \sum_{n=1}^{\infty} \sigma_{5}(n) q^{n}+504^{2} \sum_{n=2}^{\infty} \sum_{k=1}^{n-1} \sigma_{5}(k) \sigma_{5}(n-k) q^{n}+\ldots \\
& =: \sum_{n=0}^{\infty} \alpha_{n} q^{n} .
\end{aligned}
$$

Since $\sigma_{k}(n)$ is divisible by 3 when $n$ is congruent to 2 modulo 3 , as we noted in $(2.15)$, we can easily see that

$$
\alpha_{n} \equiv 0\left(\bmod 3^{3}\right) \quad \text { if } n \equiv 2(\bmod 3) \text {. }
$$

Proof of (f). To show that $\alpha_{n} \equiv 0\left(\bmod 7^{2}\right)$ when $n \equiv 4(\bmod 8)$, we need to calculate $\sigma_{5}(8 y+4)$ :

$$
\sigma_{5}(8 y+4)=\sigma_{5}\left(2^{2}\right) \sigma_{5}(2 y+1)=\left(1+2^{5}+2^{10}\right) \sigma_{5}(2 y+1) \equiv 0(\bmod 7) .
$$

This implies that $\alpha_{n} \equiv 0\left(\bmod 7^{2}\right)$ when $n \equiv 4(\bmod 8)$.

4. Coefficients of $1 / P$. We prove the congruence for the coefficients of $q^{n}$ for $1 / P(q)$.

TheOREM 4.1. Set $1 / P(q)=\sum_{n=0}^{\infty} \alpha_{n} q^{n},|q|<1$. Then

$$
\alpha_{n} \equiv 0\left(\bmod 3^{4}\right) \quad \text { for } n \equiv 2(\bmod 3) \text {. }
$$

Proof. For sufficiently small $|q|$, from (1.1), we take the geometric series expansion of $1 / P(q)$. Then

$$
\begin{aligned}
\frac{1}{P(q)}= & +24 \sum_{n=1}^{\infty} \sigma(n) q^{n}+24^{2} \sum_{n=2}^{\infty} \sum_{k=1}^{n-1} \sigma(k) \sigma(n-k) q^{n} \\
& +24^{3} \sum_{n=3}^{\infty} \sum_{k=2}^{n-1} \sum_{k_{1}=1}^{k-1} \sigma\left(k_{1}\right) \sigma\left(k-k_{1}\right) \sigma(n-k) q^{n}+\ldots
\end{aligned}
$$

So, for $n \equiv 2(\bmod 3)$,

$$
\begin{aligned}
\alpha_{n} \equiv & 3 \cdot 8 \sigma(n)+3^{2} \cdot 8^{2} \sum_{k=1}^{n-1} \sigma(k) \sigma(n-k) \\
& +3^{3} \cdot 8^{3} \sum_{k=2}^{n-1} \sum_{k_{1}=1}^{k-1} \sigma\left(k_{1}\right) \sigma\left(k-k_{1}\right) \sigma(n-k)\left(\bmod 3^{4}\right) .
\end{aligned}
$$


By $(2.25),(2.26),(2.15)$ and $(4.1)$, we see that for $n \equiv 2(\bmod 3)$,

$$
\alpha_{n} \equiv 3 \cdot 8\left(10 \sigma_{3}(n)+3(1-4 n) \sigma(n)\right)+3^{2} \cdot 8\left(7 \sigma_{5}(n)+\sigma(n)\right)\left(\bmod 3^{4}\right) .
$$

So we only need to show that for $n \equiv 2(\bmod 3)$,

$$
\begin{aligned}
8\left(10 \sigma_{3}(n)+3(1-4 n) \sigma(n)\right) & \equiv 0\left(\bmod 3^{3}\right), \\
7 \sigma_{5}(n)+\sigma(n) & \equiv 0\left(\bmod 3^{2}\right) .
\end{aligned}
$$

Since $n \equiv 2(\bmod 3)$, it has at least one prime factor $p$ that is congruent to 2 modulo 3 and whose power $r$ in $n$ is odd. Furthermore, the number of such prime factors must be odd since $n \equiv 2(\bmod 3)$. Suppose that there are more than two prime factors of $n$ that are congruent to 2 modulo 3 and with powers in $n$ that are odd. Then the congruence (4.2) can be achieved easily by $(2.1)$ and $(2.2)$, since $\sigma_{k}(n)$ is multiplicative. So we can suppose that there is only one prime factor $p \equiv 2(\bmod 3)$ whose power in $n$ is odd. Let $n=p^{r}(3 N+1)$, where $p=3 x-1, r$ is odd, and $N$ is nonnegative. By substituting $p^{r}(3 N+1)$ for $n$ in (4.2), we obtain

$$
\begin{aligned}
& 8\left(10 \sigma_{3}(n)+3(1-4 n) \sigma(n)\right) \equiv 8\left(10 \sigma_{3}\left(p^{r}\right) \sigma_{3}(3 N+1)\right. \\
& \left.+3\left(1-4 p^{r}(3 N+1)\right) \sigma\left(p^{r}\right) \sigma(3 N+1)\right)\left(\bmod 3^{3}\right) .
\end{aligned}
$$

We replace $p$ by $3 x-1$ and simplify it using (2.1). Then (4.4) is equivalent to

$$
\begin{aligned}
8\left(10 \sigma_{3}(n)+3(1-4 n) \sigma(n)\right) \equiv & 8\left(10 \sigma_{3}\left((3 x-1)^{r}\right) \sigma_{3}(3 N+1)\right. \\
& \left.+15 \sigma\left((3 x-1)^{r}\right) \sigma(3 N+1)\right)\left(\bmod 3^{3}\right) .
\end{aligned}
$$

By (2.3) and (2.21), we see that (4.5) is equivalent to

$$
\begin{aligned}
40\left(2 \cdot 3^{2} x \cdot a_{3} \widehat{A}_{3}+3 \cdot 3 x \cdot a_{1} \widehat{A}_{1}\right) & \\
& \equiv 40 \cdot 3^{2} x\left(2 a_{1} \widehat{A}_{1}+a_{1} \widehat{A}_{1}\right) \equiv 0\left(\bmod 3^{3}\right),
\end{aligned}
$$

since $\widehat{A}_{3}=\widehat{A}_{1}$. By (4.4)-(4.6), the congruence (4.2) is derived. In a similar way, we can show (4.3). Thus the proof of Theorem 4.1 is complete.

5. Coefficients of $P^{2} / R$. In this section, we prove the congruence for the coefficients of $q^{n}$ for $P^{2}(q) / R(q)$.

Theorem 5.1. Set $P^{2}(q) / R(q)=\sum_{n=0}^{\infty} \alpha_{n} q^{n},|q|<1$. Then

$$
\alpha_{n} \equiv 0\left(\bmod 3^{5}\right) \quad \text { for } n \equiv 2(\bmod 3) \text {. }
$$

Proof. As we did in the previous sections, for sufficiently small $|q|$, from (1.1) and (1.3), we take the geometric series expansion of $P^{2}(q) / R(q)$, 


$$
\begin{aligned}
\frac{P^{2}(q)}{R(q)}= & \left(1-48 \sum_{n=1}^{\infty} \sigma(n) q^{n}+24^{2} \sum_{n=2}^{\infty} \sum_{k=1}^{n-1} \sigma(k) \sigma(n-k) q^{n}\right) \\
& \times\left(1+504 \sum_{n=1}^{\infty} \sigma_{5}(n) q^{n}+504^{2} \sum_{n=2}^{\infty} \sum_{k=1}^{n-1} \sigma_{5}(k) \sigma_{5}(n-k) q^{n}+\ldots\right) \\
= & \left(1-48 \sum_{n=1}^{\infty} \sigma(n) q^{n}+48 \sum_{n=1}^{\infty}\left(5 \sigma_{3}(n)-(6 n-1) \sigma(n)\right) q^{n}\right) \\
& \times\left(1+504 \sum_{n=1}^{\infty} \sigma_{5}(n) q^{n}+504^{2} \sum_{n=2}^{\infty} \sum_{k=1}^{n-1} \sigma_{5}(k) \sigma_{5}(n-k) q^{n}+\ldots\right),
\end{aligned}
$$

where the last step is obtained by $(2.25)$. Then, for $n \equiv 2(\bmod 3)$,

$$
\begin{aligned}
\alpha_{n} \equiv & -48 \sigma(n)+48\left(5 \sigma_{3}(n)-(6 n-1) \sigma(n)\right)+504 \sigma_{5}(n) \\
& +504^{2} \sum_{k=1}^{n-1} \sigma_{5}(k) \sigma_{5}(n-k)-48 \cdot 504 \sum_{k=1}^{n-1} \sigma(k) \sigma_{5}(n-k) \\
& +48 \cdot 504 \sum_{k=1}^{n-1}\left(5 \sigma_{3}(k)-(6 k-1) \sigma(k)\right) \sigma_{5}(n-k)\left(\bmod 3^{5}\right) \\
\equiv & 48\left(5 \sigma_{3}(n)-6 n \sigma(n)\right)+504 \sigma_{5}(n)+504^{2} \sum_{k=1}^{n-1} \sigma_{5}(k) \sigma_{5}(n-k) \\
& +48 \cdot 504 \sum_{k=1}^{n-1} 5 \sigma_{3}(k) \sigma_{5}(n-k) \\
& -48 \cdot 504 \sum_{k=1}^{n-1} 6 k \sigma(k) \sigma_{5}(n-k)\left(\bmod 3^{5}\right) .
\end{aligned}
$$

By (2.27), (2.28), and (5.1), we see that

$$
\begin{aligned}
\alpha_{n} \equiv & 2^{4} \cdot 3\left(5 \sigma_{3}(n)-6 n \sigma(n)\right)+2^{3} \cdot 3^{2} \cdot 7 \sigma_{5}(n) \\
& +2^{6} \cdot 3^{4} \cdot 7^{2} \sum_{k=1}^{n-1} \sigma_{5}(k) \sigma_{5}(n-k)+2^{3} \cdot 3\left(11 \sigma_{9}(n)-3 \cdot 7 \sigma_{5}(n)\right. \\
& \left.+2 \cdot 5 \sigma_{3}(n)\right)-2^{5} \cdot 3^{2}\left(5 n \sigma_{7}(n)-2 \cdot 3 n^{2} \sigma_{5}(n)+n \sigma(n)\right)\left(\bmod 3^{5}\right) .
\end{aligned}
$$

By (2.31) and simplification, we see that

$$
\begin{aligned}
\alpha_{n} \equiv & -2^{6} \cdot 3^{2} \cdot 5 \cdot 7 \sigma(n)+2^{5} \cdot 3^{2} \cdot 439 n \sigma(n)-2^{4} \cdot 3 \cdot 5 \cdot 439 \sigma_{3}(n) \\
& +2^{4} \cdot 3^{3} \cdot 7^{2} \sigma_{5}(n)-2^{5} \cdot 3^{3} \cdot 7^{2} n \sigma_{5}(n)+2^{6} \cdot 3^{3} n^{2} \sigma_{5}(n) \\
& +2^{6} \cdot 3^{2} \cdot 5 \cdot 7 \sigma_{7}(n)-2^{5} \cdot 3^{2} \cdot 5 n \sigma_{7}(n)+2^{3} \cdot 3 \cdot 11 \sigma_{9}(n)\left(\bmod 3^{5}\right) .
\end{aligned}
$$


By (2.33), we see that

$$
\begin{aligned}
\alpha_{n} \equiv & \left(-2^{3} \cdot 3 \cdot 5 \cdot 13^{2}+2^{5} \cdot 3^{2} \cdot 449 n-2^{7} \cdot 3^{3} \cdot 5 \cdot n^{2}+2^{8} \cdot 3^{3} \cdot 5 n^{3}\right. \\
& \left.-2^{8} \cdot 3^{4} n^{4}\right) \sigma(n)+\left(-2^{4} \cdot 3 \cdot 5 \cdot 449+2^{5} \cdot 3^{3} \cdot 5^{2} n-2^{7} \cdot 3^{4} \cdot 5 \cdot n^{2}\right. \\
& \left.+2^{8} \cdot 3^{3} \cdot 5 n^{3}\right) \sigma_{3}(n)+\left(2^{8} \cdot 3^{2} \cdot 7-2^{5} \cdot 3^{2} \cdot 7 \cdot 11 n-2^{6} \cdot 3^{5} n^{2}\right) \sigma_{5}(n) \\
& +\left(2^{5} \cdot 3 \cdot 5 \cdot 37+2^{8} \cdot 3^{3} \cdot 5 n\right) \sigma_{7}(n)\left(\bmod 3^{5}\right) .
\end{aligned}
$$

We use (2.32) to obtain the equivalent congruence

$$
\begin{aligned}
\alpha_{n} \equiv & \left(2^{2} \cdot 3 \cdot 11+2^{2} \cdot 3^{2} n+2^{3} \cdot 3^{3} n^{2}+2 \cdot 3^{3} n^{3}+2 \cdot 3^{4} n^{4}\right) \sigma(n) \\
& +\left(2^{2} \cdot 3 \cdot 11+3^{4} n-3^{4} n^{2}+2 \cdot 3^{3} n^{3}\right) \sigma_{3}(n) \\
& +\left(2 \cdot 3^{2} \cdot 5+3^{2} \cdot 23 n\right) \sigma_{5}(n)+3 \cdot 7 \sigma_{7}(n)\left(\bmod 3^{5}\right) .
\end{aligned}
$$

Since $n \equiv 2(\bmod 3)$, terms with a factor of $3^{4} \sigma(n), 3^{3} \sigma_{3}(n), 3^{4} \sigma_{5}(n)$ and $3^{4} \sigma_{7}(n)$ cancel by (2.15). Next, setting $n=3 k-1$ everywhere, expanding all powers of $3 k-1$, and using (2.15), we find that

$$
\alpha_{n} \equiv(24+9 n) \sigma(n)+24 \sigma_{3}(n)+(63+18 n) \sigma_{5}(n)+21 \sigma_{7}(n)\left(\bmod 3^{5}\right) .
$$

Therefore, when $n \equiv 2(\bmod 3)$,

$$
\alpha_{n} \equiv 3\left\{(8+3 n) \sigma(n)+8 \sigma_{3}(n)+(21+6 n) \sigma_{5}(n)+7 \sigma_{7}(n)\right\}\left(\bmod 3^{5}\right) .
$$

So we only need to show that

$$
(8+3 n) \sigma(n)+8 \sigma_{3}(n)+(21+6 n) \sigma_{5}(n)+7 \sigma_{7}(n) \equiv 0\left(\bmod 3^{4}\right) .
$$

Since $n \equiv 2(\bmod 3)$, it has at least one prime factor $p$ that is congruent to 2 modulo 3 and whose power $r$ in $n$ is odd. Furthermore, the number of such prime factors must be odd since $n \equiv 2(\bmod 3)$. Suppose that there are more than three prime factors of $n$ that are congruent to 2 modulo 3 and with powers in $n$ that are odd. Then the congruence (5.2) can be achieved easily by $(2.1)$, since $\sigma_{k}(n)$ is multiplicative. So we can suppose that there are at most three prime factors congruent to 2 modulo 3 whose powers in $n$ are odd. Let $n=p^{r} p_{1}^{r_{1}} \ldots p_{m}^{r_{m}}$. We consider two cases: (i) there are exactly three primes $p, p_{1}, p_{2} \equiv 2(\bmod 3)$ whose powers $r, r_{1}, r_{2}$ in $n$ are odd, (ii) there is only one prime $p \equiv 2(\bmod 3)$ whose power $r$ in $n$ is odd. We use $a_{k}, b_{k}, c_{k}, \widehat{A}_{k}, \widehat{B}_{k}$, and $\widehat{C}_{k}$ as defined in Section 2 .

CASE (i): $n=p^{r} p_{1}^{r_{1}} \ldots p_{m}^{r_{m}}$, where $p=3 x-1, r$ is odd, and $p_{i}=3 x_{i}-1$, $r_{i}$ is odd for $i=1,2$. Let $p_{1}^{r_{1}} \ldots p_{m}^{r_{m}}=3 N+1$. By substituting $p^{r}(3 N+1)$ for $n$ in (5.2), the congruence becomes

$$
\begin{aligned}
\left(8+3 p^{r}+3^{2} p^{r} N\right) \sigma(n)+8 \sigma_{3}(n)+\left(21+6 p^{r}\right. & \left.+18 p^{r} N\right) \sigma_{5}(n) \\
& +7 \sigma_{7}(n) \equiv 0\left(\bmod 3^{4}\right)
\end{aligned}
$$

which is equivalent to

$$
8 \sigma(n)+8 \sigma_{3}(n)+7 \sigma_{7}(n) \equiv 0\left(\bmod 3^{4}\right),
$$


since $\sigma_{k}(n)$ is multiplicative and $\sigma_{k}\left(p^{r}\right), \sigma_{k}\left(p_{1}^{r_{1}}\right)$ and $\sigma_{k}\left(p_{2}^{r_{2}}\right)$ are divisible by 3 by (2.1). Furthermore, $\sigma_{3}\left(p^{r}\right)$ is divisible by $3^{2}$ by $(2.2)$. So, we see that $\sigma_{3}(n) \equiv 0\left(\bmod 3^{4}\right)$. Hence, $(5.3)$ is equivalent to

$$
\begin{aligned}
8 \sigma(n)+7 \sigma_{7}(n)= & 8 \sigma\left(p^{r}\right) \sigma(3 N+1)+7 \sigma_{7}\left(p^{r}\right) \sigma_{7}(3 N+1) \\
\equiv & 8 \sigma\left(p^{r}\right)\left(\widehat{A}_{1}+3 \widehat{B}_{1}+3^{2} \widehat{C}_{1}\right) \\
& +7 \sigma_{7}\left(p^{r}\right)\left(\widehat{A}_{7}+3 \widehat{B}_{7}+3^{2} \widehat{C}_{7}\right)\left(\bmod 3^{4}\right),
\end{aligned}
$$

where $\widehat{A}_{k}, \widehat{B}_{k}$, and $\widehat{C}_{k}$ are defined by (2.18)-(2.20). We see that $\widehat{A}_{k}$ and $\widehat{B}_{k}$, $k=1,7$, are zero since $p_{1} \equiv p_{2} \equiv 2(\bmod 3)$ and $r_{1} \equiv r_{2} \equiv 1(\bmod 2)$. By (2.3), we see that (5.4) is equivalent to

$$
8 \cdot 3^{3} x a_{1} \widehat{C}_{1}+7^{2} \cdot 3^{3} x a_{7} \widehat{C}_{7} \equiv 3^{3} x a_{1}\left(8+7^{3}\right) \widehat{C}_{1} \equiv 0\left(\bmod 3^{4}\right),
$$

since $a_{1}=a_{7}$ and $\widehat{C}_{1}=7 \widehat{C}_{7}$ by $(2.23)$.

CASE (ii): $n=p^{r} p_{1}^{r_{1}} \ldots p_{m}^{r_{m}}$, where $p=3 x-1$ and $r$ is odd. Let $p_{1}^{r_{1}} \ldots p_{m}^{r_{m}}$ $=3 N+1$. Then, by substituting $p^{r}(3 N+1)$ for $n,(5.2)$ becomes

$$
\begin{aligned}
& \left(8+3 p^{r}+3^{2} p^{r} N\right) \sigma\left(p^{r}\right) \sigma(3 N+1)+8 \sigma_{3}\left(p^{r}\right) \sigma_{3}(3 N+1) \\
& +\left(21+6 p^{r}+18 p^{r} N\right) \sigma_{5}\left(p^{r}\right) \sigma_{5}(3 N+1)+7 \sigma_{7}\left(p^{r}\right) \sigma_{7}(3 N+1) \equiv 0\left(\bmod 3^{4}\right),
\end{aligned}
$$

which, by (2.17), is equivalent to

$$
\begin{aligned}
\left(8+3 p^{r}+3^{2} p^{r} N\right) \sigma & \left.p^{r}\right)\left(\widehat{A}_{1}+3 \widehat{B}_{1}+3^{2} \widehat{C}_{1}\right) \\
& +8 \sigma_{3}\left(p^{r}\right)\left(\widehat{A}_{3}+3 \widehat{B}_{3}+3^{2} \widehat{C}_{3}\right) \\
& +\left(21+6 p^{r}+18 p^{r} N\right) \sigma_{5}\left(p^{r}\right)\left(\widehat{A}_{5}+3 \widehat{B}_{5}+3^{2} \widehat{C}_{5}\right) \\
& +7 \sigma_{7}\left(p^{r}\right)\left(\widehat{A}_{7}+3 \widehat{B}_{7}+3^{2} \widehat{C}_{7}\right) \equiv 0\left(\bmod 3^{4}\right) .
\end{aligned}
$$

By (2.21)-(2.23), congruence (5.5) is equivalent to

$$
\begin{aligned}
& \left\{\left(8+3 p^{r}\right) \sigma\left(p^{r}\right)+8 \sigma_{3}\left(p^{r}\right)+\left(21+6 p^{r}\right) \sigma_{5}\left(p^{r}\right)+7 \sigma_{7}\left(p^{r}\right)\right\} \widehat{A}_{1} \\
+ & \left\{\left(8+3 p^{r}\right) \sigma\left(p^{r}\right)+3 \cdot 8 \sigma_{3}\left(p^{r}\right)+5\left(21+6 p^{r}\right) \sigma_{5}\left(p^{r}\right)+7^{2} \sigma_{7}\left(p^{r}\right)\right\} 3 \widehat{B}_{1} \\
+ & \left\{\left(8+3 p^{r}\right) \sigma\left(p^{r}\right)+3^{2} \cdot 8 \sigma_{3}\left(p^{r}\right)+5^{2}\left(21+6 p^{r}\right) \sigma_{5}\left(p^{r}\right)+7^{3} \sigma_{7}\left(p^{r}\right)\right\} 3^{2} U \\
+ & \left\{\left(8+3 p^{r}\right) \sigma\left(p^{r}\right)+3 \cdot 8 \sigma_{3}\left(p^{r}\right)+5\left(21+6 p^{r}\right) \sigma_{5}\left(p^{r}\right)+7^{2} \sigma_{7}\left(p^{r}\right)\right\} 3^{2} V \\
+ & 3^{2} p^{r} N\left\{\sigma\left(p^{r}\right)+2 \sigma_{5}\left(p^{r}\right)\right\} \widehat{A}_{1} \equiv 0\left(\bmod 3^{4}\right) .
\end{aligned}
$$

To show (5.6), we examine carefully each expression in curly brackets in (5.6). Since $p=3 x-1$, we see that

$$
p^{r} \equiv-1+3 r x\left(\bmod 3^{2}\right) .
$$


By (2.3) we see that

$$
\left(8+3 p^{r}\right) \sigma\left(p^{r}\right)+8 \sigma_{3}\left(p^{r}\right)+\left(21+6 p^{r}\right) \sigma_{5}\left(p^{r}\right)+7 \sigma_{7}\left(p^{r}\right)
$$

$$
\begin{aligned}
\equiv & \left(5+3^{2} r x\right)(3 x)\left(a_{1}+3 b_{1}+3^{2} c_{1}\right)+8 \cdot 3^{2} x\left(1-3 x+3 x^{2}\right)\left(a_{3}+3 b_{3}+3^{2} c_{3}\right) \\
& +\left(15+2 \cdot 3^{2} r x\right)(15 x)\left(1-6 x+18 x^{2}\right)\left(a_{5}+3 b_{5}+3^{2} c_{5}\right) \\
& +7^{2} \cdot 3 x\left(1-9 x+45 x^{2}\right)\left(a_{7}+3 b_{7}+3^{2} c_{7}\right)\left(\bmod 3^{4}\right) .
\end{aligned}
$$

By (2.4)-(2.6), after reducing some coefficients modulo $3^{4}$, we see that $(5.7)$ is equivalent to

$$
\begin{aligned}
27 x\left(2+x^{2}\right) \frac{r+1}{2}+ & 27 x^{2}(2 r+1) \frac{r+1}{2}-27 x^{2} \frac{r^{2}-1}{4} \\
& +\left(5+7^{4}\right) 3^{3} x u+\left(5+7^{3}\right) 3^{3} x v \\
\equiv & 27 x\left(2+x^{2}\right) \frac{r+1}{2}+27 x^{2} \frac{(r+1)(3 r+3)}{4} \\
\equiv & 0\left(\bmod 3^{4}\right),
\end{aligned}
$$

since $x\left(2+x^{2}\right) \equiv 0(\bmod 3)$.

We next examine the coefficient of $3 \widehat{B}_{1}$ in (5.6). By the congruence $p^{r} \equiv$ $-1+3 r x\left(\bmod 3^{2}\right)$ and $(2.3)-(2.5)$, we see that

$$
\begin{aligned}
& (5.9) \quad\left(8+3 p^{r}\right) \sigma\left(p^{r}\right)+3 \cdot 8 \sigma_{3}\left(p^{r}\right)+5\left(21+6 p^{r}\right) \sigma_{5}\left(p^{r}\right)+7^{2} \sigma_{7}\left(p^{r}\right) \\
& \equiv\left(5+3^{2} r x\right)(3 x)\left(a_{1}+3 b_{1}+3^{2} c_{1}\right)+3^{3} \cdot 8 x\left(1-3 x+3 x^{2}\right)\left(a_{3}+3 b_{3}+3^{2} c_{3}\right)
\end{aligned}
$$$$
+5^{2}\left(15+2 \cdot 3^{2} r x\right)(3 x)\left(1-6 x+18 x^{2}\right)\left(a_{5}+3 b_{5}+3^{2} c_{5}\right)
$$$$
+7^{3} \cdot 3 x\left(1-9 x+45 x^{2}\right)\left(a_{7}+3 b_{7}+3^{2} c_{7}\right)
$$

$$
\equiv 15 x a_{1}+3^{2} \cdot 5 x b_{1}+3^{2} \cdot 5^{3} x a_{5}+3 \cdot 7^{3} x a_{7}+3^{2} \cdot 7^{3} x b_{7} \equiv 9 x a_{1}\left(\bmod 3^{3}\right) \text {. }
$$

Using the congruence $p^{r} \equiv-1+3 r x\left(\bmod 3^{2}\right),(2.3)$, and $(2.4)$, we find that the coefficient of $3^{2} U$ in $(5.6)$ is

$$
\begin{aligned}
& (5.10) \quad\left(8+3 p^{r}\right) \sigma\left(p^{r}\right)+3^{2} \cdot 8 \sigma_{3}\left(p^{r}\right)+5^{2}\left(21+6 p^{r}\right) \sigma_{5}\left(p^{r}\right)+7^{3} \sigma_{7}\left(p^{r}\right) \\
& \equiv\left(5+3^{2} r x\right)(3 x)\left(a_{1}+3 b_{1}+3^{2} c_{1}\right)+3^{4} \cdot 8 x\left(1-3 x+3 x^{2}\right)\left(a_{3}+3 b_{3}+3^{2} c_{3}\right)
\end{aligned}
$$$$
+5^{3}\left(15+2 \cdot 3^{2} r x\right)(3 x)\left(1-6 x+18 x^{2}\right)\left(a_{5}+3 b_{5}+3^{2} c_{5}\right)
$$$$
+7^{4} \cdot 3 x\left(1-9 x+45 x^{2}\right)\left(a_{7}+3 b_{7}+3^{2} c_{7}\right)
$$

$\equiv 15 x a_{1}+3 \cdot 7^{4} x a_{7} \equiv 0\left(\bmod 3^{2}\right)$.

By (5.9), we see that the coefficient of $3^{2} V$ in (5.6) is

$$
\begin{aligned}
\left(8+3 p^{r}\right) \sigma\left(p^{r}\right)+ & 3^{2} \cdot 8 \sigma_{3}\left(p^{r}\right) \\
& +5^{2}\left(21+6 p^{r}\right) \sigma_{5}\left(p^{r}\right)+7^{3} \sigma_{7}\left(p^{r}\right) \equiv 0\left(\bmod 3^{2}\right)
\end{aligned}
$$


We examine the coefficient of the last term in (5.6). By (2.3), we see that

$$
\begin{aligned}
3^{2} p^{r} N\left(\sigma\left(p^{r}\right)+2 \sigma_{5}\left(p^{r}\right)\right) & \equiv 9 p^{r} N\left(3 x a_{1}+30 x a_{5}\right) \\
& \equiv 27 x a_{1} N\left(\bmod 3^{4}\right) .
\end{aligned}
$$

By combining (5.8)-(5.12), we see that (5.6) is equivalent to

$$
3^{3} x a_{1} \widehat{B}_{1}+3^{3} x a_{1} \widehat{A}_{1} N \equiv 0\left(\bmod 3^{4}\right) .
$$

By (2.8), (2.9), (2.12), (2.13), and (2.19), we see that (5.13) is equivalent to

$$
3^{3} x a_{1} \widehat{A}_{1}\left(3 \sum_{j=1}^{m_{1}} \frac{-r_{j}}{2} x_{j}+3 \sum_{j=m_{1}+1}^{m} \frac{r_{j}}{2} x_{j}\right) \equiv 0\left(\bmod 3^{4}\right),
$$

where $m_{1}$ is the number of $p_{i} \equiv 2(\bmod 3)$ in $3 N+1$, and $\widehat{A}_{1} r_{j} / 2$ is an integer since $\widehat{A}_{1}=\prod_{j=m_{1}+1}^{m}\left(r_{j}+1\right)$.

This then completes the proof of (5.6) and hence also of (5.2) in Case (ii). The proof of Theorem 5.1 is thus complete.

\section{References}

[1] B. C. Berndt and P. R. Bialek, On the power series coefficients of certain quotients of Eisenstein series, submitted for publication.

[2] B. C. Berndt, P. Bialek, and A. J. Yee, Formulas of Ramanujan for the power series coefficients of certain quotients of Eisenstein series, Internat. Math. Res. Notices 2002, no. 21, 1077-1109.

[3] J. G. Huard, Z. M. Ou, B. K. Spearman, and K. S. Williams, Elementary evaluation of certain convolution sums involving divisor functions, in: Number Theory for the Millennium, Vol. 2, M. A. Bennett et al. (eds.), A K Peters, Natick, MA, to appear.

[4] D. B. Lahiri, On Ramanujan's function $\tau(n)$ and the divisor function $\sigma(n)-I$, Bull. Calcutta Math. Soc. 38 (1946), 193-206.

[5] - On Ramanujan's function $\tau(n)$ and the divisor function $\sigma_{k}(n)-I I$, ibid. 39 (1947), 33-52.

[6] S. Ramanujan, Congruence properties of partitions, Math. Z. 9 (1921), 147-153.

[7] —, Collected Papers, Cambridge Univ. Press, Cambridge, 1927; reprinted by Chelsea, New York, 1962; reprinted by Amer. Math. Soc., Providence, RI, 2000.

Department of Mathematics

University of Illinois

1409 West Green Street

Urbana, IL 61801, U.S.A.

E-mail: berndt@math.uiuc.edu yee@math.uiuc.edu 